\title{
A Set-Point-Generator for Indirect-Force-Controlled Manipulators Operating Unknown Constrained Mechanisms
}

\author{
Ewald Lutscher and Gordon Cheng \\ Institute for Cognitive Systems (www.ics.ei.tum.de) \\ Technische Universität München
}

\begin{abstract}
In this paper we propose a set-point-generator $(S P G)$ for indirect-force-controlled $(I F C)$ manipulators, interacting with mechanisms, which impose narrow bilateral kinematic constraints on their end effector, like doors, cabinets and drawers. These mechanisms could also have dynamic properties, due to their inertia, friction and gravity, which demands to consider applied forces when choosing a set-point for the IFC. Neither the type of the constraint (linear, circular), nor required manipulation forces are assumed to be known.

The proposed SPG consists of two parts: i) estimating the single direction of possible motion based on filtering of the measured end effector velocities; and ii) choosing an appropriate set-point for the underlying IFC, resulting in an effective operation of the mechanism. A major aspect of our approach is to explore the kinematic constraints with the manipulators desired set-point, avoiding direct force control, as the required interaction force is unknown and hence there is no definite reference force.
\end{abstract}

The presented approach is a generalization of our previous work on constrained manipulation of unknown mechanisms and extends the applicability to a wider class of manipulators by considering joint-level IFC and taking into account the applied forces in yielding a robust and effective controller.

The approach is evaluated in various experiments on a manipulator, providing joint space compliance.

\section{INTRODUCTION}

Direct force control is one way of controlling contact tasks without accurate position information. However, if the required forces are unknown or subject to changes, which is the case for many mechanisms in human environment, it is hard to find an appropriate reference force. Indirect force control schemes (IFC) control the interaction, as the name implies, indirectly in terms of a certain relationship between desired and actual manipulator configuration. The most popular approach among these schemes is impedance control, formulated by Hogan in his seminal paper [1], but also a simple PD-controller with feedforward compensation of the gravitational forces (PD+) can be interpreted as an IFC, provided that the gains are low enough to realize compliant behavior.

Since then, considerable works have been published on contact tasks for IFC robots, where the focus has mainly been on the generation of a certain force at the end effector. Early work of Lasky and Hsia proposes an impedanceset-point controller, capable of tracking a desired force for unknown environmental stiffness [2]. Seraji and Colbaugh present two force tracking schemes within the impedance control framework [3]. Jung et. al. present an adaptive impedance controller, also tracking force in contact with
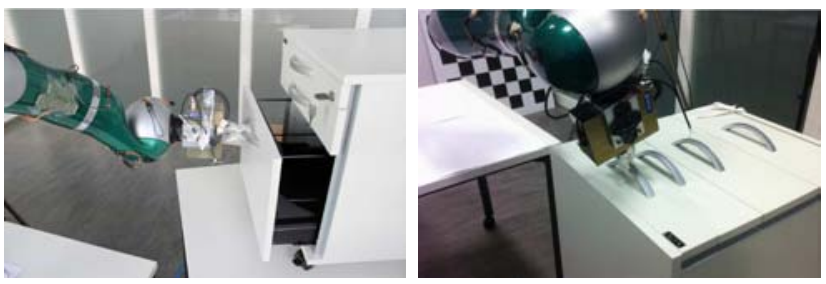

(a) kinematic constraint, linear trajectory (b) kinematic and dynamic constraint
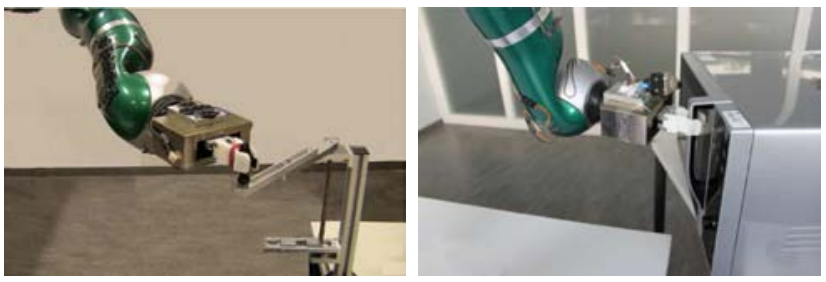

(c) circular trajectory

(d) changing required interaction forces

Fig. 1. Constraint manipulation tasks in human environment

an unknown environment [4]. These force tracking schemes are in particular interesting for industrial applications where the robot is in contact with a workpiece (e.g. deburring, grinding). In service robotics, often the actual applied forces are of less importance than the fulfillment of a certain task, like opening/closing a door or drawer.

Such constraint manipulation tasks are of major importance in the context of domestic robots and were addressed by many authors. Related works on constrained manipulation in human environment often regard it as a planning problem, e.g. [5], [6], [7], [8]. These approaches require in general specific knowledge about the constraints, imposed by the manipulated objects, which makes them prone to modeling errors and uncertainties. A general framework for manipulation under physical constraints is presented in [9], merging a Kalman filter based constraint estimator and hybrid force/position control. However, the assumption of separable velocity- and force-controllable subspaces cannot be guaranteed, when operating in human environment, e.g. spring loaded doors. Jain et al. address the particular task of pulling open doors and drawers by setting an appropriate equilibrium point for the manipulator and using a prosthetic hook as end effector [10]. They made specific assumptions regarding the type of the constrained trajectory (linear/circular) and the required forces. Special hardware devices and additional sensors (e.g. tactile modules) are utilized as well, for example in [11], to realize physical compliance or gain additional knowledge about the constraint. In [12] and [13] compliance 
was used to push a door open. In [14] a simple but effective strategy for opening an unknown door is presented, utilizing Cartesian force commands and implying knowledge about the required interaction forces. Summarizing, one can state, existing approaches either lack enough generality and robustness, do not consider required interaction forces or restrict the applicability to a small class of manipulators. Especially the fact, that required interaction forces are neglected is surprising, as many mechanisms in human environment have dynamic properties. This problem is captured in [15], where kinematic and dynamic properties of constraints are modeled in a unifying framework. This work however, focuses on the modeling aspect of the problem and does not address model uncertainties, nor any real-world applications.

In [16], we proposed an interaction controller for an admittance controlled mobile manipulator combined with a constraint estimator, based on filtering of the end effector velocity. The present work is a generalization of this approach to a wider class of robots. While the strategy developed in [16] was specific to admittance controlled robots, we formulate a more general method, which should be realizable on any IFC manipulator. Simple IFCs, like the PD+ controller, provide compliance at joint level, hence the Cartesian positioning error is not aligned with the applied end effector forces. The actual misalignment depends on the manipulator configuration, its kinematics and the joint controller gains, respectively virtual joint stiffness. For the kinematics of our KUKA-LWR, this misalignment can be easily larger than $50^{\circ}$ for equal stiffness in every joint, even for descent values ( $>0.1$ ) of the manipulability $\mu=\sqrt{\operatorname{det} \boldsymbol{J} \boldsymbol{J}^{T}}$, with $\boldsymbol{J}$ as the manipulator Jacobian (see Equation (1)). This misalignment is also often neglected by many authors working with compliant joint controllers, e.g. [10].

In the present paper, we propose a specific constrained manipulation approach for manipulators with joint-level IFC. However, the application for Cartesian compliance is straight forward and its effectiveness has already been demonstrated in [16]. We do not assume detailed knowledge neither about the constraints, nor the required interaction forces. Especially the unknown forces make it hard to apply force tracking schemes. So, instead of tracking desired forces directly, we probe the manipulators workspace with its desired configuration, while continuously estimating the current direction of motion in Cartesian space.

To our best knowledge, robotic implementations utilizing IFC in this context, was first used in [10] and our own work presented in [16]. However, interaction forces were not explicitly considered in neither of these earlier works.

\section{THEORETICAL BACKGROUND}

\section{A. Manipulator Representation}

The very basics of robotic manipulation are assumed to be known, hence only the relations directly in connection to the present work are summarized. The configuration of a manipulator with $N$ degrees of freedom (DoF) is described by a set of $N$ generalized coordinates $\boldsymbol{q}$, which are for revolute joints usually the joint angles. The base Jacobian $\boldsymbol{J}(\boldsymbol{q})$ relates the six-dimensional generalized end effector velocity or twist $\boldsymbol{v}$ to the joint velocities $\dot{\boldsymbol{q}}$ in the instantaneous kinematics

$$
\boldsymbol{v}=\left(\begin{array}{c}
\dot{p} \\
\omega
\end{array}\right)=\boldsymbol{J}(\boldsymbol{q}) \dot{\boldsymbol{q}}
$$

with $\dot{\boldsymbol{p}}$ and $\boldsymbol{\omega}$ being three-dimensional vectors representing the translational and rotational velocity of the end effector.

Another useful property of $\boldsymbol{J}$ is, that its transpose relates the end effector forces $\boldsymbol{f}$ and moments $\boldsymbol{m}$, both threedimensional, to joint torques

$$
\boldsymbol{\tau}=\boldsymbol{J}^{T} \boldsymbol{h}
$$

where $\boldsymbol{h}=\left(\begin{array}{ll}\boldsymbol{f} & \boldsymbol{m}\end{array}\right)^{T}$ is called the end effector wrench.

\section{B. Indirect Force Control}

We consider all control architectures, which establish a static relationship between the deviation of the manipulators actual configuration from the desired one and the applied forces or torques, utilizing a virtual stiffness matrix $\boldsymbol{K}_{P}$. This relationship can be either established at the end effector or at joint level, where joint-level compliance is in general easier to realize, since it does not necessarily require force/torque sensors. Examples of such control architectures are impedance type control or stiffness control, but the simplest variant of an IFC is probably a PD-controller with compensation of the gravitational forces:

$$
\boldsymbol{\tau}_{\mathrm{cmd}}=\boldsymbol{K}_{P} \boldsymbol{q}_{e}-\boldsymbol{K}_{D} \dot{\boldsymbol{q}}+\boldsymbol{g}(\boldsymbol{q})
$$

where $\boldsymbol{K}_{P}$ and $\boldsymbol{K}_{D}$ are $N \times N$ diagonal matrices, $\boldsymbol{g}(\boldsymbol{q})$ are the torques to compensate gravitational effects and $\boldsymbol{q}_{e}=\boldsymbol{q}_{d}-\boldsymbol{q}$, with $\boldsymbol{q}_{d}$ as the desired manipulator configuration. The general stability and robustness of most IFC schemes was shown in multiple publications, e.g. [17], [18]. With $\boldsymbol{q}_{d}$, the SPG indirectly sets a static interaction torque equal to $\boldsymbol{K}_{P} \boldsymbol{q}_{e}{ }^{1}$.

Fig. 2 depicts the simplified control scheme for an IFCbased architecture. If the SPG is part of the closed loop system (dotted line) the system can potentially become instable even though the IFC and the SPG are stable on their own. To overcome this in the present work, we require the SPG having a big enough time constant for being regarded as a quasi-static element, so that the feedback can be neglected.

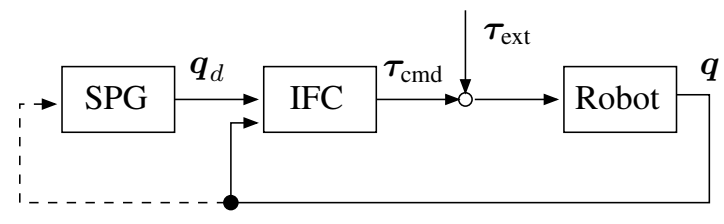

Fig. 2. Simplified IFC control scheme

\footnotetext{
${ }^{1}$ Since the IFC gives us only an interface to the static components of $\boldsymbol{\tau}_{\mathrm{cmd}}$, only static components of forces and torques are regarded from now on without explicitly mentioning it
} 


\section{Interpretation of End Effector Constraints}

As this work has a strong practical motivation we will use certain properties of every day mechanisms and ways of interacting with them.

We assume the environment as a mechanical system with 1 DoF, which applies for many real world mechanisms like doors or drawers. Furthermore, we assume the contact between the end effector and the environment is taking place at some interaction point, which imposes bilateral constraints only on the translational DoFs of the end effector. This assumption is practically motivated, as with most types of grippers, it is hard to achieve a full mechanical coupling at the interaction point. Some grasping strategies (e.g. caging grasps [19]) even explicitely aim for relaxing the task constraints. Therefore, we assume the end effector orientation, at least locally, unconstrained. Hence, the end effector translational motion is restricted to a one-dimensional trajectory in space which is determined by the mechanism it is interacting with. The type of the predefined trajectory (linear, circular) and its characteristics (arc, direction) are unknown. Thus, for stiff environments, $\dot{\boldsymbol{p}}$ is aligned with the three-dimensional unit vector $\boldsymbol{d}_{p}$, denoting the single possible direction of motion.

\section{Orthogonal Decomposition of Interaction Forces}

An important aspect of our work is, that we consider constraints with dynamic properties, which practically means, that forces need to be applied along $\boldsymbol{d}_{p}$ to operate the mechanism. Still, we want to avoid explicit modeling of the environmental dynamics, since it is probably impossible to model every potential case. Instead, we decompose interaction forces into parallel and perpendicular components with respect to $\boldsymbol{d}_{p}$. Therefore, the commanded interaction force $\boldsymbol{f}_{\text {cmd }}$, generated by the IFC, can be orthogonally decomposed according to

$$
\begin{aligned}
\boldsymbol{f}_{\mathrm{cmd}} & =\boldsymbol{f}_{\mathrm{cmd} \perp}+\boldsymbol{f}_{\mathrm{cmd} \|} \\
\boldsymbol{f}_{\mathrm{cmd} \|} & =\boldsymbol{P}_{p} \boldsymbol{f}_{\mathrm{cmd}} \\
\boldsymbol{f}_{\mathrm{cmd} \perp} & =\left(\boldsymbol{I}_{3}-\boldsymbol{P}_{p}\right) \boldsymbol{f}_{\mathrm{cmd}} .
\end{aligned}
$$

The projection matrix $\boldsymbol{P}_{p}=\boldsymbol{d}_{p} \boldsymbol{d}_{p}^{T}$ maps any threedimensional vector on $\boldsymbol{d}_{p}$ and $\boldsymbol{I}_{3}$ is the $3 \times 3$ identity matrix. The goal of the SPG is to select a set point $\boldsymbol{q}_{d}$, which leads to a static force component along $\boldsymbol{d}_{p}$, while erroneous forces, perpendicular to $\boldsymbol{d}_{p}$, should be avoided as much as possible.

\section{Set-Point-Generator For JoInt-Level-IFC}

Our SPG output is based on a simple estimator for $\boldsymbol{d}_{p}$. We employ the same technique as in [16], which will be recapitulated in section III-A before the SPG strategy for a joint-level IFC is presented in section III-B.

\section{A. Local Constraint Estimation}

As proposed by [14] in their approach of following the path of least resistance, we are applying a filter to obtain an estimate of $\boldsymbol{d}_{p}$, denoted as $\hat{\boldsymbol{d}}_{p}$. Unlike [14], we are filtering the translational Cartesian velocity $\dot{\boldsymbol{p}}$. For this, we are using a simple moving average filter, which is the unweighted mean of the previous $n$ measurements, where $n$ denotes the order of the filter. For a discrete filter, one can write

$$
\hat{\boldsymbol{d}}_{p}=\operatorname{norm}\left(\frac{1}{n} \sum_{k=0}^{n} \dot{\boldsymbol{p}}[i-k]\right)
$$

where $\dot{\boldsymbol{p}}[i]$ is the end effector translational velocity measurement at time step $i$. The normalization

$$
\operatorname{norm}(\boldsymbol{x})=\frac{\boldsymbol{x}}{\|\boldsymbol{x}\|}
$$

brings $\hat{\boldsymbol{d}}_{p}$ to unit length. In addition, we define a deadband with a threshold $v_{\text {th }}$ for the estimator. If $|\dot{\boldsymbol{p}}|<v_{\text {th }}$, the measurement is dropped and some constant vector is assigned to $\dot{\boldsymbol{p}}$, e.g. along some end effector axis or the last valid estimate. The main purpose of this action is to reject small, hence noisy velocity measurements from the estimate, but also to initialize the movement along some known direction when no motion is present. This is important, as at the beginning of the manipulation, only small, noisy motions would be detected, leading to oscillations and potential failure of the task. This local estimate allows us to track different trajectories without having to fit a curve or knowing the type (circular, linear, etc.) of the constraint trajectory. The smaller $n$ is chosen, the faster the estimation will react to changes of the actual direction. However, the consequential negative effects on stability and increased volatility of the estimate require a conservative choice for $n$. This results in a big time constant for the SPG, fulfilling the stability requirements from section II-B.

\section{B. Set Point Selection}

Using the estimated direction of possible motion $\hat{\boldsymbol{d}}_{p}$ from Equation (3), the set point is selected incrementally for every $\Delta T=t[i+1]-t[i]$ to "explore" the constraint, in order to accumulate a static force along $\hat{\boldsymbol{d}}_{p}$. As end effector orientation is assumed locally unconstrained, the desired, normalized wrench direction is $\left[\begin{array}{ll}\hat{\boldsymbol{d}}_{p} & \mathbf{0}\end{array}\right]^{T}$. Due to (2) the according joint space position increment per time step, leading to a force increment in Cartesian space, is

$$
\boldsymbol{\delta} \boldsymbol{q}_{\text {exp }}=\boldsymbol{K}_{P}^{-1} \boldsymbol{J}^{T}\left(\begin{array}{c}
\kappa \hat{\boldsymbol{d}}_{p} \\
\mathbf{0}
\end{array}\right) \Delta T
$$

where $\kappa>0$ is the desired slope of the force along $\hat{\boldsymbol{d}}_{p}$, regulating the speed of the exploration and having the unit $N / s$. From (4) we can obtain the estimated direction in joint space with

$$
\hat{\boldsymbol{d}}_{q}=\operatorname{norm}\left(\boldsymbol{\delta} \boldsymbol{q}_{\exp }\right)
$$

The according $N \times N$ projection matrix is $\boldsymbol{P}_{q}=\hat{\boldsymbol{d}}_{q} \hat{\boldsymbol{d}}_{q}^{T}$.

The set point at $t[i+1]$ is finally obtained with

$$
\boldsymbol{q}_{d}[i+1]=\boldsymbol{q}[i]+\boldsymbol{P}_{q} \boldsymbol{q}_{e}[i]+\boldsymbol{\delta} \boldsymbol{q}_{\exp }[i],
$$

where the second term of Equation (5) maps the applied static torques on $\hat{d}_{q}$, effectively canceling out $\boldsymbol{f}_{\mathrm{cmd} \perp}$. This term is of major importance, since $\hat{\boldsymbol{d}}_{q}$ changes permanently with proceeding estimation of $\hat{\boldsymbol{d}}_{p}$, but also with changing 
TABLE I

MANipUlation PARAmETER

\begin{tabular}{|l|l|}
\hline LWR sampling interval & $0.002 s$ \\
Virtual joint stiffness & $\boldsymbol{K}_{P}=40 \boldsymbol{I}_{7} \mathrm{Nm} / \mathrm{rad}$ \\
Order of Moving-Average filter & $n=500$ \\
Deadband threshold & $v_{\text {th }}=0.01 \mathrm{~m} / \mathrm{s}$ \\
Exploration speed & $\kappa=9 \mathrm{~N} / \mathrm{s}$ \\
\hline
\end{tabular}

manipulator configuration, due to the dependency of (4) on $\boldsymbol{J}(\boldsymbol{q})$.

\section{EXPERIMENTAL RESULTS}

The experiments have been carried out on our KUKA LWR-IV lightweight arm. The manipulator was running a joint space impedance controller, which details can be found in [20]. The important parameters and their default values for the experiments are listed in Table I. The values were chosen heuristically, to obtain satisfactory results in terms of execution time, stability and robustness.

All graphs in this section show the estimation error angle $\epsilon$ between $\boldsymbol{d}_{p}$ and $\hat{\boldsymbol{d}}_{p}$ in black and the static component of the applied erroneous static forces $\left\|\boldsymbol{f}_{\mathrm{cmd}_{\perp}}\right\|$ in red. The norm of the measured erroneous interaction forces (computed from joint torques) is plotted in dashed magenta.

The first set of experiments is a comparison between our approach and a Cartesian trajectory-following strategy, both using the same estimation method from Section III-A. The Cartesian SPG uses inverse kinematics to move the virtual end effector along $\hat{\boldsymbol{d}}_{p}$ and projecting its desired position on the line, defined by the current end effector position and $\hat{\boldsymbol{d}}_{p}$, using the orthogonal projector $\boldsymbol{P}_{p}$ from Section II-D. The Cartesian exploration speed was chosen to provide a roughly similar performance in terms of execution time as the joint space SPG.

The task was to pull up a $1.2 \mathrm{~kg}$ mass for $0.3 \mathrm{~m}$. The motion of the mass was restricted to a linear trajectory. This setup was realized by a drawer in a vertical configuration (see Fig. 1b). The graphs in Fig. 3 show the data for our approach and the trajectory-following strategy, each with different initial errors. It can be clearly seen, that for the trajectory-following strategy, where interaction forces are not considered, erroneous forces remain, even for very small estimation errors. This is due to the misalignment of the Cartesian position deviation and the applied static forces for joint space compliance. In our approach, for large initial estimation errors, erroneous forces build up, until the mechanism starts moving and a descent estimation is obtained. After this, the erroneous forces vanish rapidly. Note also, that forces build up linearly for the proposed method according to $\kappa$.

For the measurements in Fig. 4, the drawer is in a horizontal configuration (see Fig. 1a), imposing a nearly pure kinematic constraint, with only small forces required. Also, in this case our approach performs better in terms of applied erroneous forces, even though not as clearly as in the dynamically constrained case.

To test our approach also for circular trajectories and evaluate its robustness, we performed an experiment in turning a crank with varying radius and $\kappa$ (see Fig. 1c). As can be seen from the left graphs in Fig. 5, our approach was successful, even for very small radii (please also see the attached video). However, one observes increasing estimation errors when shorten the radius. From the right graphs in Fig. 5, which show data for turning a crank with radius $17 \mathrm{~cm}$ and varying the values for $\kappa$, it can be seen, how increased exploration speed leads to larger estimation errors and erroneous forces. This comes from the delay, introduced by the filter (3), highlighting the major disadvantage of this simple estimation method.

Our approach also proved its robustness when we manually disturbed the manipulation procedure or manipulated devices with changing required forces, e.g. microwave door in Fig. 1d (this can be seen in the attached video).

\section{CONCLUSiOn AND Future WORK}

We presented a set-point-generator for indirect-forcecontrolled manipulators in order to operate kinematically and dynamically constrained mechanisms. Neither a model of the mechanism, nor specific knowledge about the required interaction forces are assumed, which makes the method quite versatile. We also make only very general demands on the manipulator, performing the task. Experiments have shown the robustness and generality of the proposed controller for various mechanisms.

\section{ACKNOWLEDGEMENTS}

This work is supported in part within the DFG excellence initiative research cluster Cognition for Technical Systems CoTeSys (www. cotesys.org).

\section{REFERENCES}

[1] N. Hogan, "Impedance control: An approach to manipulation," in American Control Conference, 1984. IEEE, 1984, pp. 304-313.

[2] T. Lasky and T. Hsia, "On force-tracking impedance control of robot manipulators," Proceedings. 1991 IEEE International Conference on Robotics and Automation, no. April, pp. 274-280, 1991.

[3] H. Seraji and R. Colbaugh, "Force Tracking in Impedance Control," The International Journal of Robotics Research, vol. 16, no. 1, pp. 97-117, Feb. 1997.

[4] S. Jung, T. Hsia, and R. Bonitz, "Force Tracking Impedance Control of Robot Manipulators Under Unknown Environment," IEEE Transactions on Control Systems Technology, vol. 12, no. 3, pp. 474-483, May 2004.

[5] K. Nagatani and S. Yuta, "Designing strategy and implementation of mobile manipulator control system for opening door," Proceedings of IEEE International Conference on Robotics and Automation, no. April, pp. 2828-2834, 1996.

[6] L. Peterson, D. Austin, and D. Kragic, "High-level control of a mobile manipulator for door opening," Proceedings. 2000 IEEE/RSJ International Conference on Intelligent Robots and Systems (IROS 2000) (Cat. No.OOCH37113), pp. 2333-2338, 2000.

[7] S. Wieland, D. Gonzalez-Aguirre, N. Vahrenkamp, T. Asfour, and R. Dillmann, "Combining force and visual feedback for physical interaction tasks in humanoid robots," in Humanoid Robots, 2009. Humanoids 2009. 9th IEEE-RAS International Conference on. IEEE, 2009, pp. 439-446.

[8] W. Meeussen, M. Wise, S. Glaser, S. Chitta, C. McGann, P. Mihelich, E. Marder-Eppstein, M. Muja, V. Eruhimov, T. Foote, and Others, "Autonomous door opening and plugging in with a personal robot," in International Conference on Robotics and Automation (ICRA), 2010 IEEE. IEEE, 2010, pp. 729-736.

[9] T. Lefebvre, J. Xiao, H. Bruyninckx, and G. de Gersem, "Active compliant motion: a survey," Advanced Robotics, vol. 19, no. 5, pp. 479-499, June 2005. 


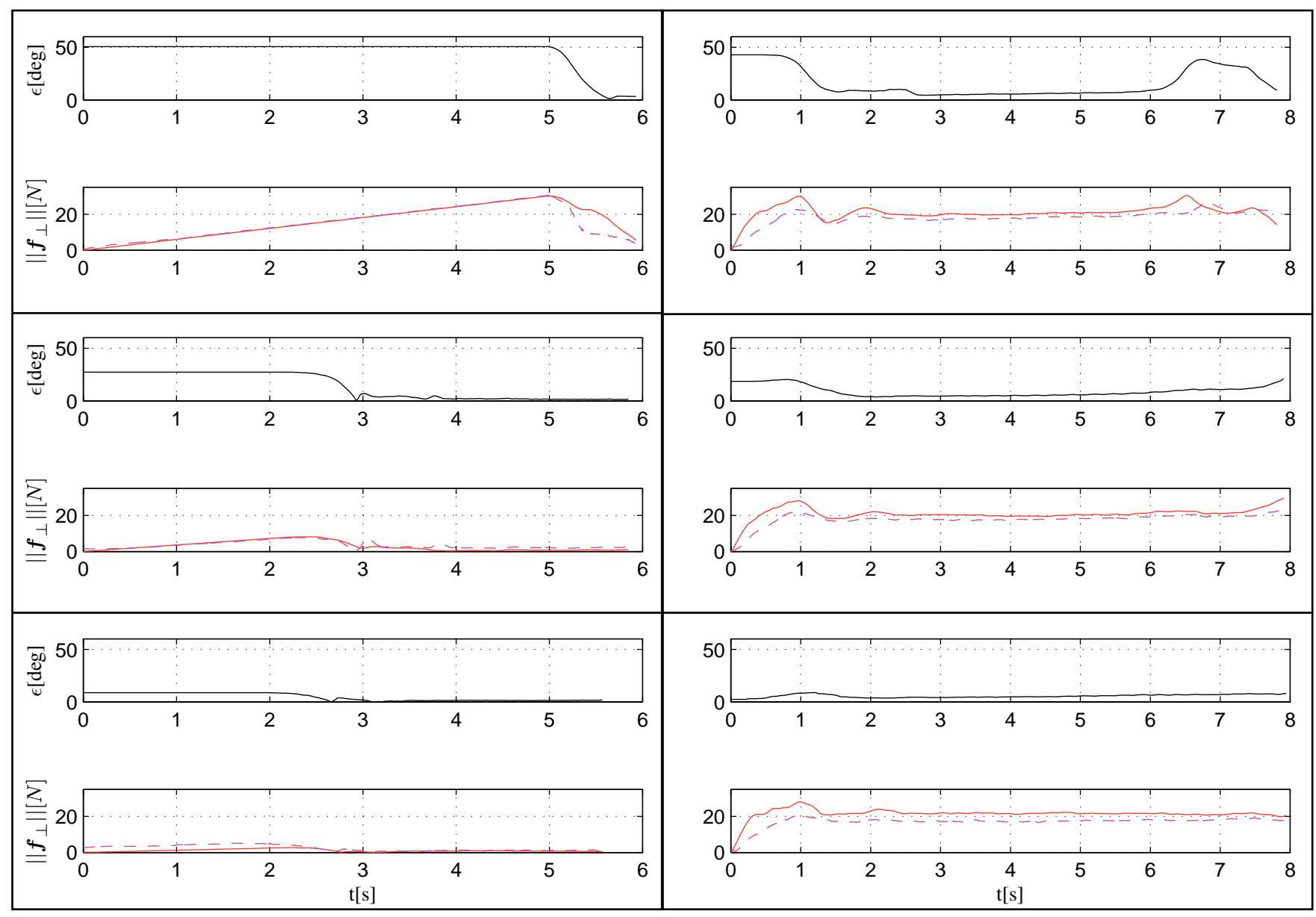

Fig. 3. Pulling up a $1.2 \mathrm{~kg}$ mass with different initial errors, using our approach (left) and a Cartesian trajectory-following approach (right)

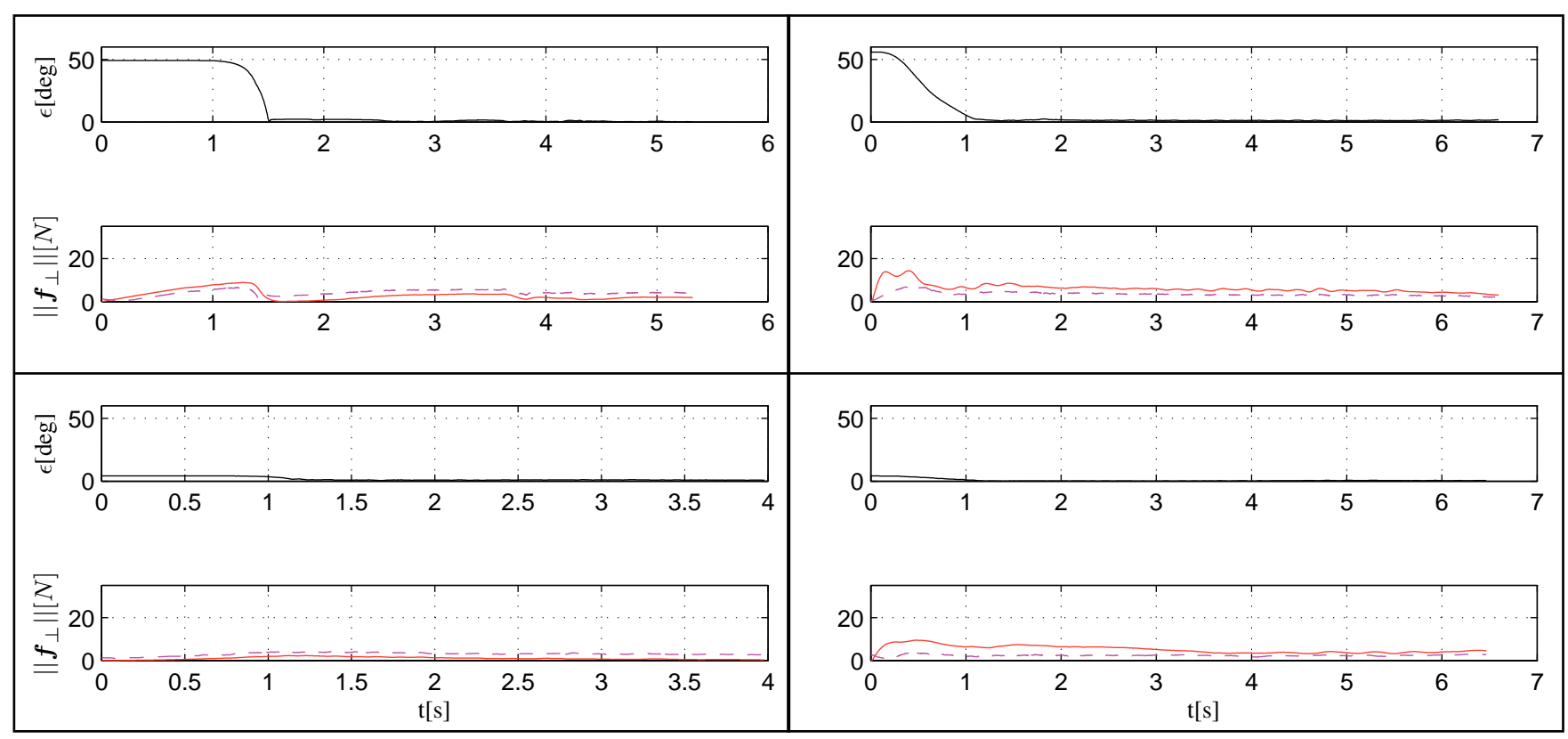

Fig. 4. Opening a drawer (pure kinematic constraint) using our approach (left) and a Cartesian trajectory-following approach (right)

[10] A. Jain and C. C. Kemp, "Pulling open novel doors and drawers with equilibrium point control," 2009 9th IEEE-RAS International Conference on Humanoid Robots, pp. 498-505, Dec. 2009.
[11] C. Rhee, M. Kim, and H. Lee, "Door opening control using the multifingered robotic hand for the indoor service robot," IEEE International Conference on Robotics and Automation, 2004. Proceedings. ICRA 


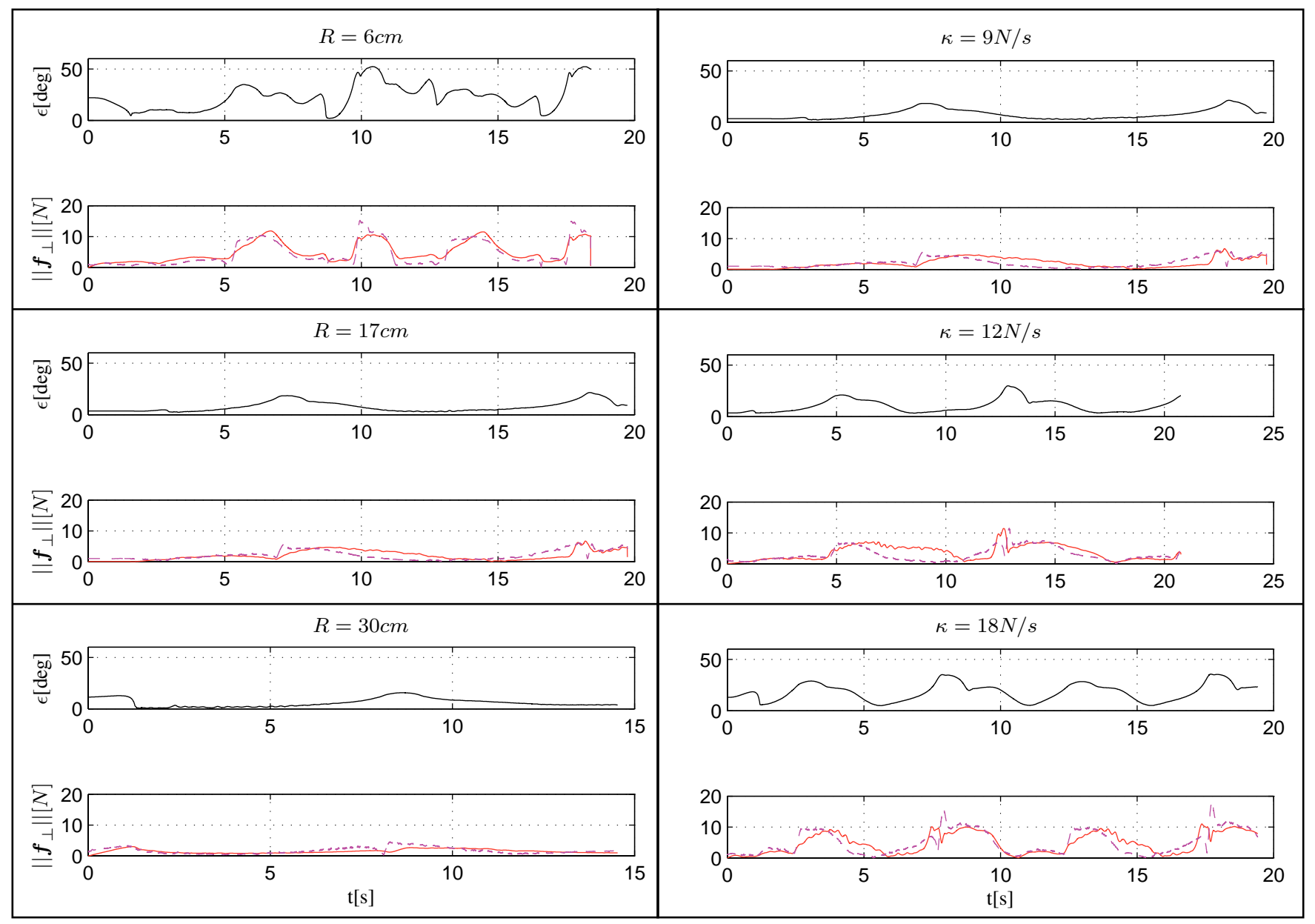

Fig. 5. Left: Turning a crank with varying radius $R$; Right: varying exploration speed $\kappa$ for $R=17 \mathrm{~cm}$

'04. 2004, pp. 4011-4016 Vol.4, 2004.

[12] C. Ott, B. Baeuml, C. Borst, and G. Hirzinger, "Autonomous opening of a door with a mobile manipulator: A case study," in 6th IFAC Symposium on Intelligent Autonomous Vehicles (IAV), 2007.

[13] C. Kessens, J. Rice, and D. Smith, "Utilizing compliance to manipulate doors with unmodeled constraints," Intelligent Robots and, pp. 483489, 2010.

[14] G. Niemeyer and J. Slotine, "A simple strategy for opening an unknown door," in Robotics and Automation, 1997. Proceedings., 1997 IEEE International Conference on, vol. 2, no. April. IEEE, 1997, pp. $1448-1453$.

[15] A. De Luca and C. Manes, "Modeling of robots in contact with a dynamic environment," Robotics and Automation, IEEE Transactions on, vol. 10, no. 4, pp. 542-548, 1994.

[16] E. Lutscher, M. Lawitzky, G. Cheng, and S. Hirche, "A control strategy for operating unknown constrained mechanisms," International Con- ference on Robotics and Automation (ICRA), 2010 IEEE, pp. 819-824, 2010.

[17] P. Tomei, "A simple PD controller for robots with elastic joints," Automatic Control, IEEE Transactions on, vol. 36, no. 10, pp. 1208$1213,1991$.

[18] N. Hogan, "On the stability of manipulators performing contact tasks," IEEE Journal on Robotics and Automation, vol. 4, no. 6, pp. 677-686, 1988.

[19] R. Diankov, S. Srinivasa, D. Ferguson, and J. Kuffner, "Manipulation planning with caging grasps," in Humanoid Robots, 2008. Humanoids 2008. 8th IEEE-RAS International Conference on. IEEE, 2008, pp. 285-292.

[20] A. Albu-Schaffer, C. Ott, and G. Hirzinger, "A unified passivity based control framework for position, torque and impedance control of flexible joint robots," Robotics Research, pp. 5-21, 2007. 